EPJ Web of Conferences 95, 01006 (2015)

DOI: $10.1051 /$ epjconf/ 20159501006

(C) Owned by the authors, published by EDP Sciences, 2015

\title{
Status of the CBM experiment
}

\author{
Johann M. Heuser ${ }^{1, a}$, on behalf of the CBM Collaboration \\ ${ }^{1}$ GSI Helmholtz Center for Heavy Ion Research GmbH, Darmstadt, Germany
}

\begin{abstract}
The Compressed Baryonic Matter (CBM) experiment at the Facility for AntiProton and Ion Research (FAIR) will explore the phase diagram of strongly interacting matter at highest net baryon densities and moderate temperatures. The CBM physics program will be started with beams delivered by the SIS 100 synchrotron, providing energies from 2 to $11 \mathrm{GeV} /$ nucleon for heavy nuclei, up to $14 \mathrm{GeV} /$ nucleon for light nuclei, and $29 \mathrm{GeV}$ for protons. The highest net baryon densities will be explored with ion beams up to $45 \mathrm{GeV} /$ nucleon energy delivered by SIS 300 in the next stage of FAIR. Collision rates up to $10^{7}$ per second are required to produce very rare probes with unprecedented statistics in this energy range. Their signatures are complex. These conditions call for detector systems designed to meet the extreme requirements in terms of rate capability, momentum and spatial resolution, and a novel DAQ and trigger concept which is not limited by latency but by throughput. The article discusses the development status of the CBM sub-systems for charged particle tracking, vertex detection, electron/muon identification, hadron/time-of-flight measurement, electromagnetic and zero-degree calorimetry, in terms of prototypes and expected physics performance. The concept and development status of CBM's central detector, the Silicon Tracking System STS are presented in somewhat more detail.
\end{abstract}

\section{Introduction}

The Compressed Baryonic Matter [1] experiment at FAIR will conduct a systematic research program to explore the phase diagram of strongly interacting matter at highest net baryon densities [2] and moderate temperatures. These conditions are to be created in collisions of heavy-ion beams with nuclear targets in the projectile beam energy range of 2 to $45 \mathrm{GeV} /$ nucleon $\left(\sqrt{s}_{N N}=1.9-9 \mathrm{GeV}\right)$, initially coming from the SIS 100 synchrotron (up to $14 \mathrm{GeV} /$ nucleon) and in a next step from SIS 300 enabling studies at the highest net baryon densities. Also proton-nucleus interactions will be studied. The accelerators are overviewed in [3]. Heavy-ion collisions at rates from $10^{5}$ to $10^{7}$ per second are required to produce very rare probes with unprecedented statistics in this energy range. Their signatures are complex and include excitation functions of yields and phase space distributions of strangeness, di-leptons, charm, and strange matter with few or no data available at FAIR energies yet. These conditions call for detector systems designed to meet the extreme requirements in terms of rate capability, momentum and spatial resolution, and a novel data acquisition and trigger concept which is not limited by latency but by throughput. The fast and radiation hard detector systems require in particular to be equipped with free-streaming read-out electronics, producing self-triggered time-stamped detector

\footnotetext{
ae-mail: J.Heuser@gsi.de
}

This is an Open Access article distributed under the terms of the Creative Commons Attribution License 4.0, which permits unrestricted use, distribution, and reproduction in any medium, provided the original work is properly cited. 
data that will be sent to a high speed data acquisition system for on-line event reconstruction in a powerful computing farm. The event determination can be considered as a 4-dimensional track finding procedure, where the fourth coordinate is the time stamp of the tracks' hits in the detectors. As event classes can only be identified after the full reconstruction of the detector data leading to the events as such, the CBM experiment has no hardware detector triggers but builds entirely on software triggers as an output of the on-line event analysis. The on-line computing will cope with the high collision and data rates through parallelization of the analyses, with suitable algorithms running on many-core processing units. An example of the challenge is CBM's central detector for the reconstruction of charged particles, the Silicon Tracking System. Figure 1 shows the simulated trajectories of about 700 charged particles, created in a single $\mathrm{Au}+\mathrm{Au}$ collision at $25 \mathrm{GeV} /$ nucleon, traversing the detector and producing numerous hits in the silicon micro-strip sensors with their projective topology. On-line event reconstruction requires their pile-up free read-out and presentation to a computing system that can process the data at those rates.

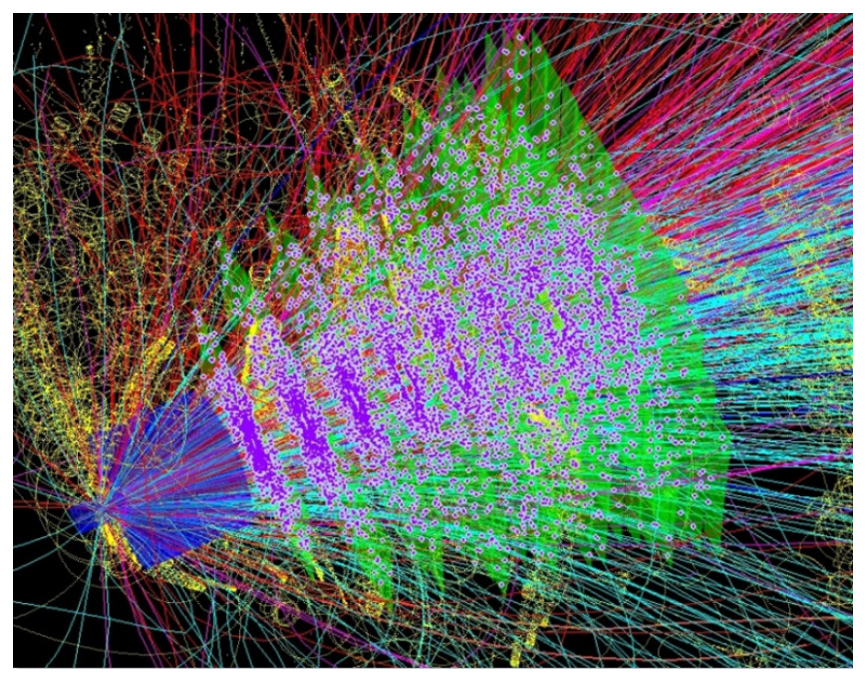

Figure 1. Particles from a central $\mathrm{Au}+\mathrm{Au}$ collision at $25 \mathrm{GeV} /$ nucleon traversing the Silicon Tracking System, with the intersection points shown as pink dots in the green tracking stations. Delta-electrons produced at the target and other places are shown in yellow color.

\section{CBM cave and detector set-up}

The CBM experiment will be set up in an underground cave to which the beams from the synchrotrons SIS 100/300 will be extracted. The cave is part of FAIR's modular start version, and will be built as the second out of five construction modules. The cave is already fully designed. Its location at FAIR and its structure are shown in Fig. 2. The underground hall hosting the detector systems is $37 \mathrm{~m}$ long, $27 \mathrm{~m}$ wide and $17 \mathrm{~m}$ heigh. The CBM detector arrangement is depicted in Fig. 3, presenting one of its configurations, here for electron-hadron measurements. The target area is surrounded by the superconducting dipole magnet hosting the Silicon Tracking System and the Micro Vertex Detector for charged particle tracking and the detection of short-lived decays. Downstream the beam follow the 
Ring Imaging Cherenkov Detector and the Transition Radiation Detector for electron identification and pion separation. The Time-of-Flight system identifies hadrons. The Projectile Spectator Detector serves event plane characterization. Two further detector systems are shown in parking position: the Muon Detection System, at the RICH position for a CBM muon program, and the Electromagnetic Calorimeter, to be used for measurements of direct photons and neutral mesons decaying into photons. The non-interacting beam particles, $99 \%$ of all with a typical target of $1 \%$ nuclear interaction length, are guided in a vacuum beam pipe through the experiment and are absorbed in the beam dump. Also shown in the hall is the HADES experiment, placed in front of the CBM experiment, that will conduct a complementary physics program in separate data taking periods. The CBM set-up is conceived as an experimental facility. Next to configuring the detectors for either electron-hadron or muon measurements the system can also be completed in steps. It may be operated in a minimal mode at FAIR start-up with only key sub-systems and the necessary part of the data acquisition and on-line computing installed, which are lateron completed. Similarly, different optimizations of the experiment to perform measurements at SIS 100 and SIS 300 are possible. This is illustrated in Fig. 4 on the example of three potential detector configurations for a muon program. The actual start-up scenario is still to be decided on by the CBM Collaboration.

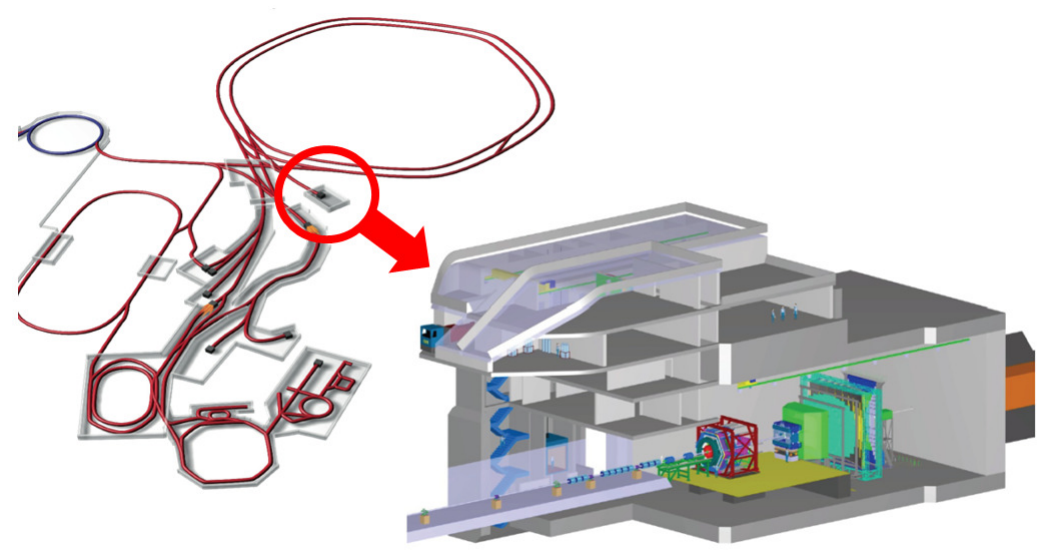

Figure 2. Location of the CBM experiment at the FAIR site. Layout of the CBM building and underground hall.

\section{Development of CBM sub-systems}

\subsection{Super-conducting dipole magnet}

The design of the super-conducting magnet realizes a field integral of $1 \mathrm{Tm}$ in the region of the Silicon Tracking System. The magnet yoke provides a gap of $140 \mathrm{~cm}$ vertical and $280 \mathrm{~cm}$ horizontal aperture. Field clamps at the downstream face limit the stray field into the RICH detector system. The magnet contains self-protecting coils made from $\mathrm{Cu} / \mathrm{SC}$ in ratio 9:1. The magnet's weight will be 140 tons. The Technical Design Report of the magnet is approved [4]. The construction in industry is forthcoming. 


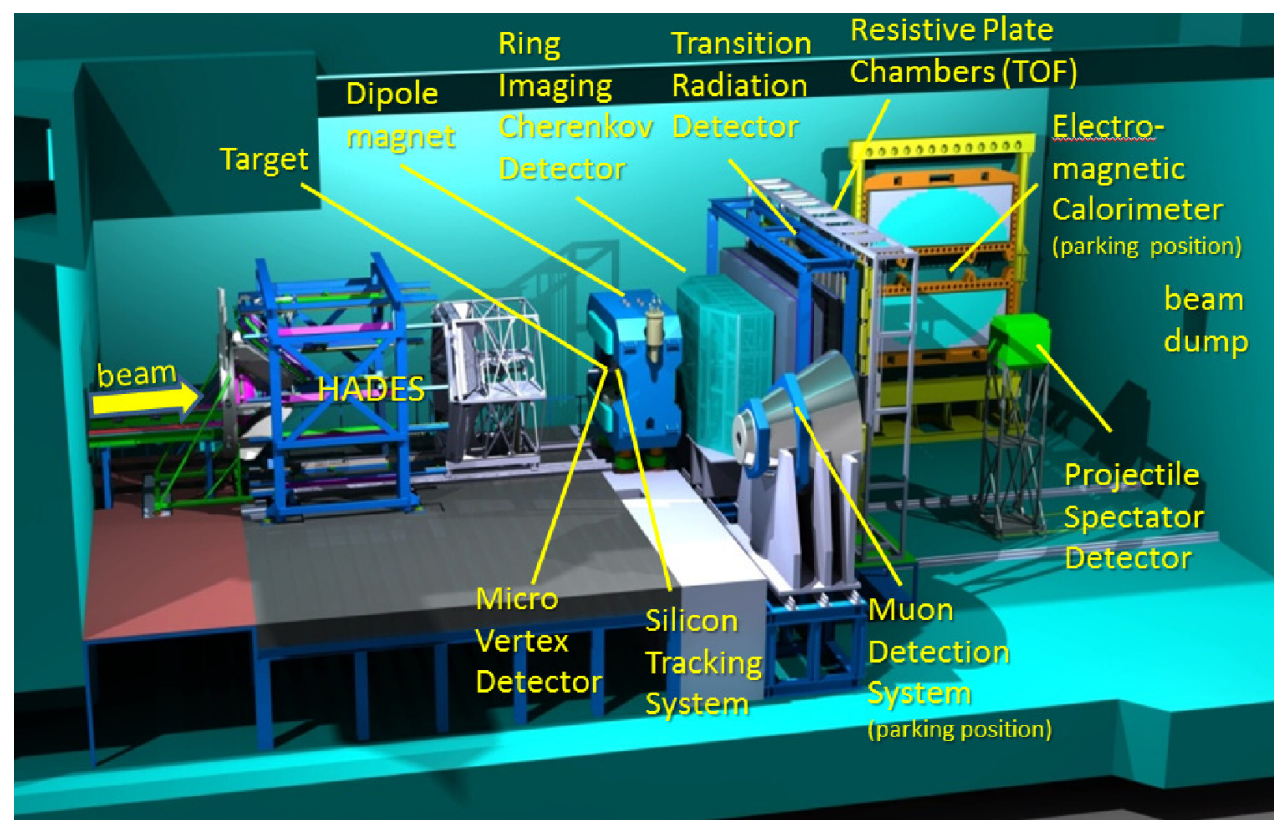

Figure 3. View into the CBM cave on the detector set-up of CBM (right) and HADES (left). The CBM experiment is shown in electron-hadron configuration.
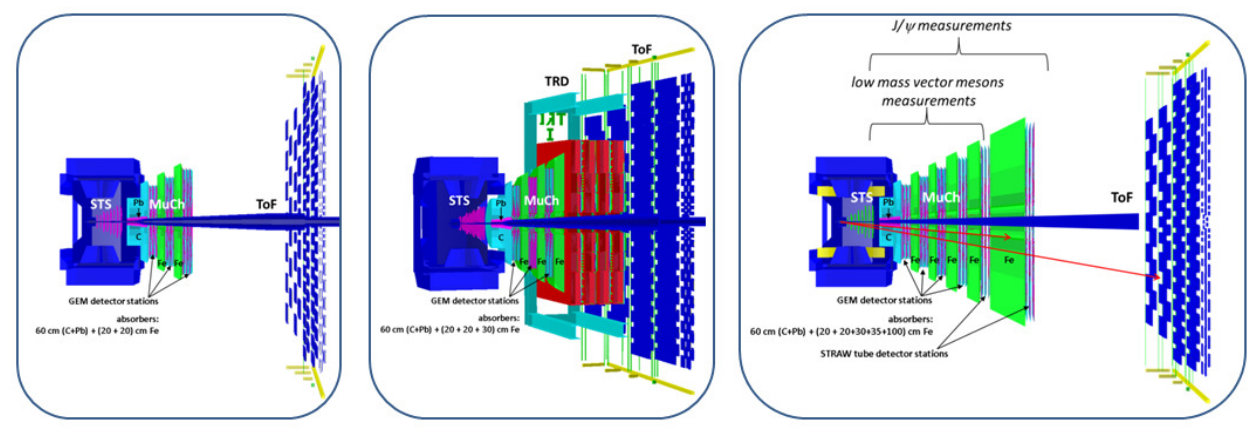

Figure 4. Three possible muon configurations: "start-up" with full STS, partial MUCH and TOF systems (left), "SIS 100" with full STS, partial MUCH, full TRD for tracking, and full TOF (middle), and "SIS 300" with full STS, MUCH and TOF systems (right).

\subsection{Silicon Tracking System}

The STS, shown in Fig. 5, performs track identification and momentum determination with eight large-aperture stations comprising double-sided silicon micro-strip detectors. The collision environment presents few charged particles in $\mathrm{p}+\mathrm{A}$ collisions at SIS 100 to hundreds of charged tracks in $\mathrm{A}+\mathrm{A}$ collisions at SIS 100 and SIS 300, at $10^{5}$ to $10^{7}$ collisions per second. Next to track reconstruc- 
tion alone, the detector is important also for the identification of decay topologies within its aperture. The STS tracks are linked to further detectors upstream (MVD) and downstream (MUCH/RICH) for refined measurements of vertices of short-lived decays and particle identification. The STS Technical Design Report [5] is approved. The development of the detector system is advanced, aiming at bringing technology developments for components and assembly procedures (see Fig. 6) and the system engineering to production readiness in 2016.
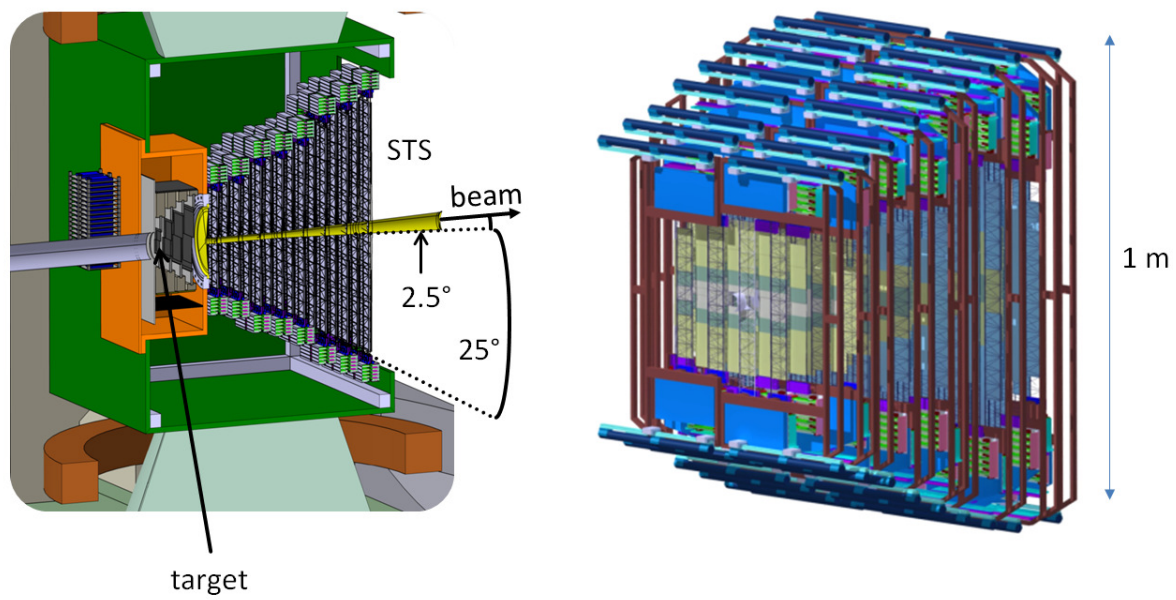

Figure 5. Conceptual design of the Silicon Tracking System, showing a cut through the detector in the dipole magnet (left). The ensemble of eight tracking stations to be mounted on the detector's main support frame (right).

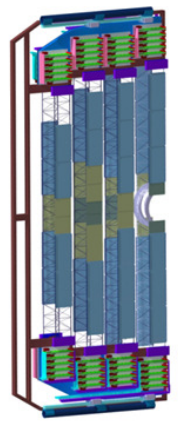

mech. unit

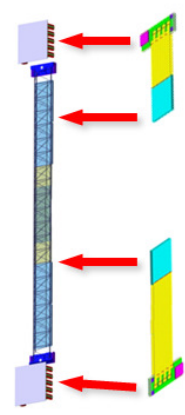

ladder modules

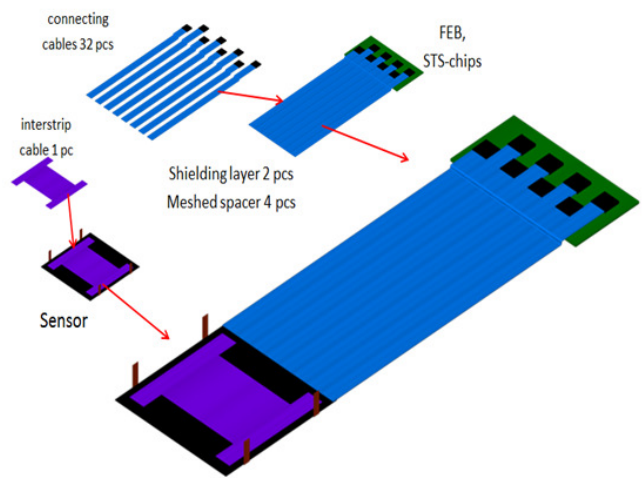

Figure 6. Concept of arranging detector modules onto ladders, and ladders onto mechanical units to build the tracking stations (left). Components of a detector module, the most complex object of the Silicon Tracking System to be assembled (right). 


\subsection{Micro Vertex Detector}

The MVD adds very high spatial resolution to the tracking in order to detect the decay products of short-lived particles, like D mesons. The detector will consist of monolithic active pixel sensors (MAPS) arranged on four stations in the target vacuum chamber [6]. The radiation tolerance of this technology is at present $10^{13} \mathrm{n}_{e q} / \mathrm{cm}^{2}$ (non-ionizing), and 3 Mrad (ionizing). The current sensor for the study of a prototype system is MIMOSA-26, comprising per chip 600k pixels that are read out with few ten $\mu \mathrm{s} /$ frame. The read-out speed limits interaction rates to about $10^{5} / \mathrm{s}$ in runs with the MVD included. The pixel size is 20 by $20 \mu \mathrm{m}^{2}$ yieldig a position resolution of $\sigma=4 \mu \mathrm{m}$. The four-station system achieves a vertex resolution of $50-100 \mu \mathrm{m}$ along the beam axis. The die is thinned to $50 \mu \mathrm{m}$. The system under development arranges the sensors on both sides of a diamond carrier equipped with flex-print circuits. The material budget of such assembly is $<0.5 \%$ radiation length.

\subsection{Ring Imaging Cherenkov Detector}

The RICH detector is designed to provide identification of electrons and suppression of pions in the momentum range below $10 \mathrm{GeV} / \mathrm{c}$. This will be achieved using a gaseous $\mathrm{RICH}$ detector build in a projective geometry with focusing mirror elements and a photo detector. The radiator gas is $\mathrm{CO}_{2}$ with a pion threshold for Cherenkov radiation of $4.65 \mathrm{GeV} / \mathrm{c}$. The RICH Technical Design Report is approved [7]. A full-size prototype has been tested in beam at the CERN PS, equipped with mirror and photo detection arrays to assess different multi-anode photo multipliers.

\subsection{Transition Radiation Detector}

Three TRD stations consisting of three detector layers each will provide particle tracking and identification of electrons and positrons with momenta exceeding $1.5 \mathrm{GeV} / \mathrm{c}$. The pion suppression factor is 10 (100) at SIS 100 (SIS 300). This is achieved with several types of radiators. Different types of readout chambers with and without (fast) drift regions have been tested in beam with read-out electronics to extract signal features.

\subsection{Muon Chamber System}

The MUCH detection system is a segmented hadron absorber instrumented with layers of tracking stations. The absorbers are made from iron and carbon. The tracking stations are triplet layers of chambers based on GEM and straw tube detectors. The MUCH system allows performing the measurements of both $\mathrm{J} / \psi$ and low-mass vector mesons. The full set-up for operation at SIS 300 comprises 4 GEM stations and 2 straw-tube stations. The absorbers are slabs of $60 \mathrm{~cm}$ carbon, with lead shields around the beam pipe, and $(20+20+30+100) \mathrm{cm}$ iron. The reduced set-up for SIS 100 consists of 3 GEM stations and absorbers from $60 \mathrm{~cm}$ carbon and $(20+20+20) \mathrm{cm}$ iron. Prototypes of GEM stations made from different GEM foil stacks and sizes have been tested in beam, including ageing studies and verification of rate capability.

\subsection{Time-Of-Flight Detector}

Hadron identification is performed through their time-of-flight measurement. The TOF wall will have an active area of about $120 \mathrm{~m}^{2}$. The detector technology is based on resistive plate chambers providing a time resolution of $60 \mathrm{ps}$. They are set up in $6 \mathrm{~m}$ (SIS100) and $10 \mathrm{~m}$ (SIS300) distance from the target. The hit rates range between 10 and $25 \mathrm{kHz} / \mathrm{cm}^{2}$ for the inner, and between 2 and $10 \mathrm{kHz} / \mathrm{cm}^{2}$ for the outer sections of the wall. Prototypes have been tested in various in-beam experiments. 


\subsection{Calorimeters}

The electro-magnetic calorimeter ECAL provides detection of photons and neutral mesons $\left(\pi_{0}, \eta\right)$ decaying into photons. The "shashlik" type calorimeter will be based on modules of 140 layers made from $1 \mathrm{~mm}$ lead and $1 \mathrm{~mm}$ scintillator stacks, in cell sizes of 3 by 3,6 by 6 and 12 by $12 \mathrm{~cm}$. The modules will be arranged in a wall or in a tower geometry with variable distances from the target. Protototypes of modules have been assembled and tested.

The participant-spectator-detector PSD serves the determination of collision centrality and orientation of an event plane. It is a fully compensating modular lead-scintillator calorimeter with high and uniform energy resolution. The detector will be built from 44 individual modules, with 60 lead/scintillator layers, facing to the beam a surface of 20 by $20 \mathrm{~cm}$. The read-out is realized by multi-anode photo detectors via wavelength shifting fibers.

\subsection{Read-out electronics, data acquisition and on-line event selection}

Several read-out ASICs are under development for the CBM detector systems. Among those is the STS-XYTER chip [8] in the Silicon Tracking System. It integrates an asynchronous front-end with self-triggering sampling of signals, and a synchronous back-end for hit de-randomization and shipping. The time-stamped data is streamed to aggregating and processing boards for time-slice sorting and a computing farm for further on-line processing. High-throughput event building and data analysis is realized by applying massive parallelization to reconstruction algorithms and analysis tasks. Steps of the event determination and event reconstruction are the formation of "pre-events" by timeslice sorting of detector hits, followed by track finding, track fitting and the assignment of tracks to interactions, the events. The last step is to identify particles, decay topologies and other signatures. Along the data processing, "software" triggers are assigned to events. Only selected data will be stored, with a reduction of the data volume by up to a factor of 1000 as compared to the raw data stream [9]. The track finding is a large combinatorial task that is well parallelizable and applicable to many-core CPU/GPU computing systems. The construction of the FAIR Tier-0 data center ("GreenIT cube") has started at GSI. It will host the CBM on-line event selector FLES. Prototypes of this computing architecture are the LOEWE CIC, Frankfurt, the "Mini Cube", GSI, and the computing facility at the FAIR Russia Research Center.

\section{Outlook and timeline}

The development of CBM's detector and technical systems is advanced [10] and will be largely completed by the end of 2016. Technical design reports for the Superconducting Dipole Magnet, the Silicon Tracking System and the Ring Imaging Cherenkov detector have already been approved by FAIR. The reports for the Time-Of-Flight system, the muon detection system MUCH and the ParticipantSpectator-Detector are currently under evaluation. Design reports for the Micro Vertex Detector, the Transition Radiation Detector and the On-line Computing System will be submitted to FAIR in 2015. The serial production of components and the construction of the systems are planned to be completed in 2019. The installation of sub-systems into the CBM cave and their commissioning without beam will follow in 2020. Experiments are expected to be started with beams from SIS 100 in 2021. The detailed schedule is to be confirmed. 


\section{References}

[1] CBM web page, http://www.fair-center.eu/for-users/experiments/cbm.html

[2] V. Friese, Heavy-Ion Physics at High Baryon Densities, these proceedings

[3] O. Kester, Status of the FAIR Project, these proceedings

[4] CBM Collaboration, Technical Design Report for the CBM Superconducting Dipole Magnet, CBM Report (October 2013), http://www.fair-center.eu/fileadmin/fair/experiments/CBM/TDR/ CBM_magnet_TDR_31_10_2013-nc.pdf

[5] CBM Collaboration, Technical Design Report for the CBM Silicon Tracking System, GSI Report 2013-4 (October 2013), http://repository.gsi.de/record/54798

[6] M. Deveaux and J. Heuser, The Silicon Detector Systems of the Compressed Baryonic Matter (CBM) experiment at FAIR, PoS(Vertex2013)009, http://pos.sissa.it/archive/conferences/198/009/Vertex2013_009.pdf

[7] CBM Collaboration, Technical Design Report for the CBM Ring Imaging Cerenkov Detector, GSI Report 2013-4 (June 2013), http://repository.gsi.de/record/65526

[8] R. Kleczek et al., Time and energy measuring front-end electronics for long silicon strip detectors readout, Proc. IEEE NSS/MIC (2013), http://dx.doi.org/10.1109/NSSMIC.2013.6829538

[9] V. Friese, Lect. Notes Comp. Sci. 7125 (2012) 17, http://link.springer.com/chapter/10.1007\%2F978-3-642-28212-6_2

[10] CBM Collaboration, CBM Progress Report 2013, https://www-alt.gsi.de/documents/DOC-2014-Mar-16-1.pdf 